\title{
INTERAÇÃo ENTRE HERBICIDAS INIBIDORES DA ACCASE E DIFERENTES Formulações de Glyphosate no Controle de Capim-Amargoso ${ }^{1}$
}

\author{
Accase and Glyphosate Diferent Formulations Herbicides Association Interactions on Sourgrass \\ Control
}

\author{
BARROSO, A.A.M. ${ }^{2}$, ALBRECHT, A.J.P. ${ }^{2}$, REIS, F.C. ${ }^{2}$ e FILHO R.V. ${ }^{2}$
}

\begin{abstract}
RESUMO - O glyphosate é um herbicida não seletivo que inibe a produção de aminoácidos aromáticos essenciais à sobrevivência de plantas. Esse herbicida é disponibilizado no mercado em distintas formulações que podem apresentar diferenças no controle de espécies. A mistura de herbicidas é uma ferramenta importante no controle de plantas daninhas resistentes, sendo uma opção à mistura de inibidores da ACCase ao herbicida glyphosate. Este trabalho contou com a aplicação de diferentes misturas de quatro graminicidas e três formulações de glyphosate em dois estádios vegetativos de plantas de capim-amargoso resistente a esse herbicida. O controle químico foi avaliado por notas visuais de controle, aos 7, 14 e 28 dias após a aplicação, e pela medida de biomassa fresca das plantas, aos 28 dias depois da aplicação. As misturas foram avaliadas como antagonísticas, sinergísticas ou aditivas, segundo o modelo proposto por Colby. Dos tratamentos utilizados, conclui-se que todos são efetivos no manejo da resistência de Digitaria insularis, com exceção do uso de glyphosate isolado, porém o controle deve ser feito em estádios iniciais de desenvolvimento da planta. Em estádios mais desenvolvidos, o melhor controle se deu na associação de quizalofop às formulações sal de amônio e sal potássico de glyphosate; contudo, outras medidas de controle, além da associação de produtos, devem ser tomadas.
\end{abstract}

Palavras-chave: graminicidas, Digitaria insularis, associação de herbicidas.

ABSTRACT - Glyphosate is a non-selective herbicide that inhibits the production of essential amino acids for plant survival. This herbicide is available in the market in different formulations that can produce different results for weed control. The mixture of herbicides is an important option to control herbicide resistant weeds, and it is an alternative to mixing ACCase inhibiting herbicides with glyphosate. This work was carried by applying different mixtures of four grass herbicides and 3 glyphosate formulations sprayed at two stages of development of sourgrass. Chemical control was evaluated by visual injury rating at 7, 14 and 28 days after application, and by measuring the fresh weight of the plants at 28 days after the application. The mixtures were classified as antagonistic, synergist or additive according to the model proposed by Colby. All the treatments were effective in the early control of sourgrass except for the application of glyphosate alone. In late applications with more developed plants, the mixture of quizalofop with glyphosate salts (ammonium and potassium) showed better control, but other control measures plus the herbicide mixture should be taken.

Keywords: grass herbicides, Digitaria insularis, herbicide association.

Recebido para publicação em 25.11.2013 e aprovado em 29.4.2014.

Universidade de São Paulo, Piracicaba-SP, Brasil, <arthuragro07@hotmail.com>.

Planta Daninha, Viçosa-MG, v. 32, n. 3, p. 619-627, 2014 


\section{INTRODUÇÃO}

O glyphosate é um herbicida foliar de aplicação em pós-emergência não seletivo que pertence ao grupo dos inibidores da síntese de aminoácidos. Sua atuação dá-se inibindo a atividade enzimática da enzima 5-enolpiruvilshiquimato-3-fosfato sintase (EPSPs) (Shaner \& Bridges, 2003). Essa inibição leva à não produção de aminoácidos aromáticos (fenilalanina, tirosina e triptofano). Esses aminoácidos são essenciais para a produção da parede celular, defesa da planta contra patógenos e insetos e produção de hormônios. Seus efeitos secundários ocorrem na respiração, fotossintese e síntese proteica da planta (Duke, 1990).

O glyphosate é o herbicida mais utilizado no mundo (Hartzler, 2008). Seu uso elevou-se nos últimos anos devido à crescente adoção de culturas transgênicas tolerantes ao herbicida e à facilidade de seu uso, pois ele apresenta baixa toxicidade, baixo custo, amplo espectro de controle e rápida adsorção no solo (Velini et al., 2009). Com seu uso contínuo, plantas resistentes a essa molécula foram selecionadas, o que aconteceu com a planta de capim-amargoso.

Além do glyphosate, o controle químico de capim-amargoso conta com poucas opções de herbicidas. O uso de herbicidas de contato, como o paraquat, é dificultado devido à formação de densas touceiras, que impedem que o herbicida atinja a planta toda, a qual geralmente rebrota. Essa rebrota acontece muitas vezes, pois o herbicida não atinge o rizoma (Melo et al., 2010).

Sobram como opções os herbicidas recomendados que são sistêmicos, sendo eles os inibidores da ACCase, os inibidores da sintese de carotenoides e inibidores da EPSPs, o que restringe a variação de uso de herbicidas aplicados em pós-emergência. Destes, apenas o glyphosate é recomendado para uso em estádios avançados do desenvolvimento da planta (Rodrigues \& Almeida, 2012).

Com relação ao glyphosate, estão disponibilizadas no mercado diferentes formulações dele, que podem apresentar diferenças no controle de espécies, devido a fatos como absorção e translocação diferenciadas (Molin \& Hirase, 2005; Li et al., 2005). Essas formulações podem comportar-se de maneira diferente se associadas a diferentes herbicidas - entre eles, os graminicidas.

Assim, muitos são os resultados produzidos na associação de produtos, porém faltam índices que comprovem o efeito de interação de diferentes mecanismos de ação e o estudo do uso de diferentes sais de glyphosate (formulações), que podem apresentar comportamentos diferentes na planta. Uma maneira de predizer esses valores dá-se pelo uso da fórmula de Colby (Colby, 1967).

Uma mistura é considerada sinergística quando seu efeito é superior à resposta prevista pelo efeito somado das aplicações isoladas de cada herbicida; aditiva, quando o efeito somado das aplicações isoladas é semelhante ao efeito esperado; e antagonística, quando o efeito da mistura é menor do que o efeito somado das aplicações individuais (Staker \& Oliver, 1998). Trabalhos relatam que a mistura de glyphosate com outros herbicidas tem resultado em interações antagônicas e sinergísticas (Lich et al., 1997).

Este trabalho teve como objetivo de avaliar o efeito das misturas de diferentes sais de glyphosate associados a diferentes graminicidas no controle de plantas de capim-amargoso resistentes ao herbicida glyphosate, em diferentes estádios fenológicos, através do estabelecimento de relações sinérgicas, aditivas ou antagonísticas das misturas.

\section{MATERIAL E MÉTODOS}

O experimento foi conduzido em casa de vegetação no ano de 2012. Sementes de Digitaria insularis provenientes da fazenda Cambuhy, localizada em Matão-SP, foram coletadas em lotes onde a aplicação de glyphosate ocorreu por mais de 10 anos consecutivos. A resistência dessa espécie foi comprovada, apresentando fator de resistência de 2,36 vezes, por meio de ensaios de doseresposta ao herbicida glyphosate (dados não apresentados). As sementes foram originadas de uma população de, pelo menos, 40 diferentes plantas.

Essas sementes foram postas para germinar em vasos com capacidade de 3 litros, utilizando como substrato um solo médio com as 
seguintes características: $\mathrm{pH}: 6,2 ; \mathrm{P}: 5 \mathrm{mg} \mathrm{kg}^{-1}$; $\mathrm{K}, \mathrm{Ca}, \mathrm{Mg}, \mathrm{H}+\mathrm{Al}, \mathrm{SB}$, T e V (\%): 4,2; 41; $15 ;<26$; 60,$1 ; 72,5 \mathrm{mmol}_{c} \mathrm{dm}^{-3}$; e $83 \%$, respectivamente; e teores de argila, silte e areia total de: 276,86 e $638 \mathrm{~g} \mathrm{~kg}^{-1}$, respectivamente.

Foram realizados dois experimentos de controle químico com as plantas originadas dessas sementes. No primeiro, as aplicações ocorreram quando as plantas apresentavam quatro folhas, e no segundo, elas estavam em perfilhamento, com 3-4 perfilhos cada. O delineamento experimental utilizado em ambos foi o inteiramente casualizado, constituído de 20 tratamentos e seis repetições no primeiro experimento e 20 tratamentos e quatro repetições no segundo. Não foram necessárias aplicações de inseticidas, fungicidas ou adubações.

Para aplicação dos tratamentos, utilizouse de uma câmara de aplicação experimental de herbicidas, acionada por um motor elétrico, com uma ponta de pulverização montada em bico leque jato plano, modelo TeeJet 8002 , pulverizando em média a $50 \mathrm{~cm}$ do alvo, com volume de calda de $200 \mathrm{~L} \mathrm{ha}^{-1}$ e pressão constante de 40 Psi. Os tratamentos utilizados podem ser verificados na Tabela 1 .

A avaliação da eficácia de controle proporcionada pela aplicação dos herbicidas deu-se através de escala visual de injúria das plantas, que varia de 0 a 100\%, aos 7, 14 e 28 dias após a aplicação dos tratamentos (DAA), em que 0\% representou a ausência de sintomas e $100 \%$, a morte total da planta, segundo o método proposto pela Sociedade Brasileira da Ciência das Plantas Daninhas (SBCPD, 1995). Aos 28 DAA, foi medida a biomassa fresca da parte aérea das plantas.

Os dados obtidos aos 28 DAA foram analisados, quanto às interações, pelo modelo proposto por Colby. A equação utilizada para cálculo da resposta esperada na interação foi a seguinte:

$$
E=100-\frac{(100-X) \times(100-Y)}{100}
$$

Tabela 1 - Nome comum, dose em litro/quilograma do produto comercial por hectare e estádio de aplicação dos tratamentos com herbicidas nas plantas de capim-amargoso

\begin{tabular}{|c|c|c|c|c|c|}
\hline \multirow{2}{*}{\multicolumn{2}{|c|}{ Tratamento }} & \multicolumn{4}{|c|}{ Dose $\left(\mathrm{L}\right.$ ou kg ha $\left.{ }^{-1}\right)$} \\
\hline & & \multicolumn{2}{|c|}{ 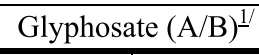 } & \multicolumn{2}{|c|}{ Graminicidas (A/B) } \\
\hline \multirow{5}{*}{$\begin{array}{c}\text { Glyphosate } \\
\text { sal de isopropilamina }\end{array}$} & - & 2,0 & 3,0 & 0,00 & 0,00 \\
\hline & Quizalofop & 2,0 & 3,0 & 0,50 & 0,50 \\
\hline & Haloxyfop & 2,0 & 3,0 & 0,50 & 0,50 \\
\hline & Sethoxydim & 2,0 & 3,0 & 1,00 & 1,25 \\
\hline & Clethodim & 2,0 & 3,0 & 0,40 & 0,45 \\
\hline \multirow{5}{*}{$\begin{array}{l}\text { Glyphosate } \\
\text { sal de amônio }\end{array}$} & - & 1,5 & 1,5 & 0,00 & 0,00 \\
\hline & Quizalofop & 1,5 & 1,5 & 0,50 & 0,50 \\
\hline & Haloxyfop & 1,5 & 1,5 & 0,50 & 0,50 \\
\hline & Sethoxydim & 1,5 & 1,5 & 1,00 & 1,25 \\
\hline & Clethodim & 1,5 & 1,5 & 0,40 & 0,45 \\
\hline \multirow{5}{*}{$\begin{array}{l}\text { Glyphosate } \\
\text { sal potássico }\end{array}$} & - & 2,8 & 2,8 & 0,00 & 0,00 \\
\hline & Quizalofop & 2,8 & 2,8 & 0,50 & 0,50 \\
\hline & Haloxyfop & 2,8 & 2,8 & 0,50 & 0,50 \\
\hline & Sethoxydim & 2,8 & 2,8 & 1,00 & 1,25 \\
\hline & Clethodim & 2,8 & 2,8 & 0,40 & 0,45 \\
\hline- & Quizalofop & 0,0 & 0,0 & 0,50 & 0,50 \\
\hline- & Haloxyfop & 0,0 & 0,0 & 0,50 & 0,50 \\
\hline- & Sethoxydim & 0,0 & 0,0 & 1,00 & 1,25 \\
\hline- & Clethodim & 0,0 & 0,0 & 0,40 & 0,45 \\
\hline- & Testemunha & 0,0 & 0,0 & 0,00 & 0,00 \\
\hline
\end{tabular}

${ }^{1 /}(\mathrm{A} / \mathrm{B})$ = A: aplicação em estádio vegetativo inicial; B: aplicação em estádio vegetativo avançado. 
em que $\mathrm{E}$ é o controle ou redução de crescimento esperado pela mistura de herbicidas; e X e Y representam a porcentagem de controle ou redução do crescimento proporcionado pelos herbicidas aplicados isoladamente. A redução de biomassa fresca (Rd) das plantas daninhas foi calculada seguindo a equação:

$$
R d=100-\frac{\text { Biomassa fresca da planta tratada }}{\text { Biomassa fresca da testemunha }} \times 100
$$

em que a biomassa fresca da testemunha foi a média dos valores obtidos na ausência da aplicação de herbicidas. Os valores calculados foram comparados com os valores observados por meio da análise de variância, com diferença mínima significativa avaliada em nível de $\mathrm{p}<0,05$. Quando houve diferença entre as médias dos tratamentos, a associação de herbicidas foi designada de antagonística, se o valor de controle resultante da mistura foi menor do que o valor esperado da soma das aplicações isoladas, e de sinergística, se o valor da associação de produtos foi superior ao valor esperado da soma das aplicações isoladas. Quando não houve diferença significativa, a associação foi considerada aditiva.

\section{RESULTADOS E DISCUSSÃO}

No tocante ao controle químico proporcionado pelos herbicidas aplicados em estádios iniciais de desenvolvimento da planta, notouse que todos os tratamentos foram efetivos (> 80\% de controle), com exceção do uso de glyphosate isolado - no caso, o uso do herbicida glyphosate sal potássico e sal de amônio. O uso de glyphosate sal de isopropilamina mostrouse eficaz no controle. Esse controle pode estar associado à presença da tecnologia Transorb ${ }^{\circledR}$ em sua formulação (MONSANTO, 2013). Outros trabalhos destacam a ação mais rápida dessa formulação no controle de plantas daninhas (Silva et al., 2011).

Com relação à redução de biomassa fresca, os dados revelam que a aplicação em estádios iniciais de desenvolvimento das plantas (3-4 folhas) é fundamental para o sucesso do manejo da espécie. Mesmo com a aplicação de glyphosate, a redução dessa massa ultrapassou valores de $90 \%$.

No entanto, no final do período avaliado, próximo aos 28 dias após a aplicação (DAA), notou-se que algumas plantas tratadas com glyphosate apresentavam rebrotas em sua base.

Aos 7 DAA, o uso de qualquer herbicida contendo glyphosate apresentou o pior controle dos tratamentos. Na comparação entre eles, os piores foram glyphosate sal de amônio e sal potássico. Dos graminicidas testados, todos tiveram o mesmo efeito, assim como as misturas destes com glyphosate. A mistura de quizalofop ou clethodim ao herbicida glyphosate foi superior ao uso isolado de glyphosate, no caso de glyphosate sal de isopropilamina, e todas as misturas foram superiores ao uso isolado de glyphosate sal de amônio e sal potássico (Tabela 2).

Aos 14 DAA, o pior controle resultou da aplicação de glyphosate sal de amônio e sal potássico. Dos graminicidas isolados, o uso de clethodim foi inferior ao uso de haloxyfop. Das misturas, todas foram mais efetivas que o uso de glyphosate isolado, exceto a mistura de glyphosate sal de isopropilamina e sethoxydim.

Aos 28 DAA, todos os tratamentos apresentaram resultados semelhantes estatisticamente, com exceção daqueles com herbicidas glyphosate sal de amônio e sal potássico aplicados isoladamente, inferiores aos demais.

$\mathrm{Na}$ redução de biomassa fresca, o uso de glyphosate sal de isopropilamina foi superior ao uso das demais formulações de glyphosate. Os graminicidas tiveram efeitos semelhantes. As misturas com quizalofop foram superiores ao uso isolado de glyphosate sal potássico; as de haloxyfop, ao uso isolado de glyphosate sal de amônio e potássico; as com sethoxydim, ao uso isolado de glyphosate sal potássico; e as de clethodim, ao uso isolado de glyphosate sal de amônio e potássico. Esse fato ocorreu devido ao menor controle proporcionado pela aplicação de glyphosate sal potássico, em comparação ao glyphosate sal de amônio (Tabela 2). Todas as misturas, assim como todos os graminicidas, resultaram em efeitos semelhantes entre si e satisfatórios na redução da biomassa de plantas de amargoso.

Como o controle alcançado pela aplicação de produtos isolados ou redução de biomassa fresca foi muito alto, seguindo a fórmula de Colby, o dividendo assumiu valores próximos 
Tabela 2 - Médias de controle visual de capim-amargoso aos 7, 14 e 28 dias após a aplicação (DAA) e porcentagem de redução de biomassa fresca em relação à testemunha, aos 28 DAA

\begin{tabular}{|c|c|c|c|c|}
\hline \multirow{2}{*}{ Tratamento } & \multicolumn{3}{|c|}{ Controle Visual (\%) } & \multirow{2}{*}{$\begin{array}{l}\text { Redução Biomassa } \\
\text { Fresca }(\%)\end{array}$} \\
\hline & $7 \mathrm{DAA}$ & $14 \mathrm{DAA}$ & $28 \mathrm{DAA}$ & \\
\hline Glyphosate $^{1 /}$ & $58,3 \mathrm{bc}$ & $85,5 \mathrm{c}$ & $98,8 \mathrm{a}$ & $98,0 \mathrm{a}$ \\
\hline Glyphosate + Quizalofop & $78,3 \mathrm{a}$ & $99,5 \mathrm{a}$ & $100,0 \mathrm{a}$ & $98,6 \mathrm{a}$ \\
\hline Glyphosate + Haloxyfop & $75,0 \mathrm{ab}$ & $99,2 \mathrm{a}$ & $100,0 \mathrm{a}$ & $96,4 \mathrm{ab}$ \\
\hline Glyphosate + Sethoxydim & $70,8 \mathrm{ab}$ & $93,7 \mathrm{abc}$ & $96,3 \mathrm{a}$ & $98,2 \mathrm{a}$ \\
\hline Glyphosate + Clethodim & $80,0 \mathrm{a}$ & $99,3 \mathrm{a}$ & $100,0 \mathrm{a}$ & $98,1 \mathrm{a}$ \\
\hline Glyphosate $^{2 /}$ & $32,5 \mathrm{~d}$ & $65,0 \mathrm{~d}$ & $68,3 \mathrm{~b}$ & $91,3 \mathrm{bc}$ \\
\hline Glyphosate + Quizalofop & $75,8 \mathrm{ab}$ & $99,3 \mathrm{a}$ & $100,0 \mathrm{a}$ & $95,6 \mathrm{ab}$ \\
\hline Glyphosate + Haloxyfop & $76,7 \mathrm{a}$ & $99,0 \mathrm{a}$ & $100,0 \mathrm{a}$ & $97,8 \mathrm{a}$ \\
\hline Glyphosate + Sethoxydim & $78,3 \mathrm{a}$ & $98,3 \mathrm{ab}$ & $100,0 \mathrm{a}$ & $96,7 \mathrm{ab}$ \\
\hline Glyphosate + Clethodim & $74,2 \mathrm{ab}$ & $98,3 \mathrm{ab}$ & $91,7 \mathrm{a}$ & $97,2 \mathrm{a}$ \\
\hline Glyphosate $^{3 /}$ & $46,7 \mathrm{~cd}$ & $72,5 \mathrm{~d}$ & $70,8 \mathrm{~b}$ & $89,3 \mathrm{c}$ \\
\hline Glyphosate + Quizalofop & $79,2 \mathrm{a}$ & $99,3 \mathrm{a}$ & $100,0 \mathrm{a}$ & $97,3 \mathrm{a}$ \\
\hline Glyphosate + Haloxyfop & $73,3 \mathrm{ab}$ & $97,3 \mathrm{ab}$ & $100,0 \mathrm{a}$ & $95,7 \mathrm{ab}$ \\
\hline Glyphosate + Sethoxydim & $72,5 \mathrm{ab}$ & $97,0 \mathrm{ab}$ & $100,0 \mathrm{a}$ & $96,6 \mathrm{ab}$ \\
\hline Glyphosate + Clethodim & $75,8 \mathrm{ab}$ & $98,2 \mathrm{ab}$ & $99,7 \mathrm{a}$ & $97,3 \mathrm{a}$ \\
\hline Quizalofop & $75,8 \mathrm{ab}$ & $98,0 \mathrm{ab}$ & $100,0 \mathrm{a}$ & $96,1 \mathrm{ab}$ \\
\hline Haloxyfop & $76,7 \mathrm{a}$ & $99,2 \mathrm{a}$ & $100,0 \mathrm{a}$ & $97,1 \mathrm{a}$ \\
\hline Sethoxydim & $70,8 \mathrm{ab}$ & $98,5 \mathrm{ab}$ & $99,2 \mathrm{a}$ & $96,6 \mathrm{ab}$ \\
\hline Clethodim & $67,5 \mathrm{ab}$ & $88,8 \mathrm{bc}$ & $95,0 \mathrm{a}$ & $95,3 \mathrm{ab}$ \\
\hline $\mathrm{DMS}^{4 / 1}$ & 17,50 & 10,03 & 12,54 & 5,67 \\
\hline $\mathrm{F}$ & $12,61 * *$ & $24,66 * *$ & $91,83 * *$ & $4,40 * *$ \\
\hline $\mathrm{CV}^{5 / 1}$ & 11,92 & 5,11 & 6,55 & 2,82 \\
\hline
\end{tabular}

1/2 Sal de isopropilamina; 는 sal de amônio; ${ }^{3}$ sal potássico; ${ }^{4}$ diferença mínima significativa; ${ }^{5}$ coeficiente de variação; adição de óleo mineral segundo recomendação do fabricante em todos os tratamentos. *, ** Médias seguidas pela mesma letra na coluna não diferem entre si pelo teste de Tukey em nível de probabilidade de erro de $5 \%$.

ou iguais a 0 ; assim, desde que o outro herbicida não prejudicasse esse alto valor atingido (o que não ocorreu nesse caso), todos os tratamentos foram considerados aditivos (análise estatística resultante não significativa) e não serão apresentados.

Quando a aplicação dos herbicidas ocorreu em outro estádio da planta (planta perfilhando), o resultado de controle não foi o mesmo. Ressalta-se que os valores das doses foram corrigidos segundo informações de bula. O controle, que sempre passava de $80 \%$, com exceção do glyphosate, em poucos casos ultrapassou esse valor aos 28 DAA. Notou-se que, com o uso de quaisquer dos sais de glyphosate, o controle foi sempre baixo. A mistura de herbicidas graminicidas melhorou, em todos os casos, o controle final atingido. $\mathrm{O}$ uso de graminicidas isoladamente também foi eficaz se comparado ao uso de glyphosate, com exceção do uso isolado de clethodim.

Quando se analisou a redução de biomassa fresca, viu-se que o clethodim foi inferior aos demais graminicidas. Sua aplicação reduziu a massa das plantas daninhas em niveis iguais ou inferiores aos niveis de redução resultantes da aplicação de glyphosate, mesmo em mistura com glyphosate sal potássico. Os melhores usos foram resultado da aplicação de quizalofop em mistura com glyphosate sal de isopropilamina ou isolado e da aplicação de haloxyfop isoladamente.

Pela análise estatística, ao final do período avaliado (28 DAA), viu-se que o pior controle foi alcançado pelo tratamento com glyphosate 
sal de amônio e depois da aplicação de glyphosate sal de isopropilamina, sal potássico e clethodim isolado. Intermediários a esses valores foram o uso de misturas de graminicidas com glyphosate e o uso dos outros graminicidas isolados.

O melhor resultado de controle foi da aplicação de glyphosate sal de amônio juntamente com quizalofop $(91,3 \%)$, glyphosate sal potássico mais quizalofop $(91,0 \%)$, quizalofop isolado $(82,5 \%)$ e clethodim em mistura com glyphosate sal de isopropilamina $(81,3 \%)$ ou sal de amônio (82,5\%). A redução de biomassa resultou em valores muito próximos uns dos outros. A redução resultante da aplicação isolada de glyphosate ou da associação deste com graminicidas foi semelhante. Dos graminicidas aplicados isoladamente, apenas o uso de haloxyfop foi superior ao uso de clethodim (Tabela 3).
Analisando os resultados segundo a fórmula de Colby e considerando-se os valores de controle e a redução de biomassa fresca, os resultados das misturas não foram tão efetivos quanto os que ocorreram com a aplicação em estádio vegetativo inicial da espécie. Quando se comparou o glyphosate sal de isopropilamina com os graminicidas, os resultados foram antagonísticos em todos os casos, com exceção do controle da mistura com clethodim, que foi aditiva. Contudo, esse fato pode ser explicado pelo baixo controle proporcionado pela aplicação isolada do herbicida clethodim. Assim, esperava-se que qualquer efeito superior a esse, até mesmo pelo uso de glyphosate, fosse aditivo ao seu uso em mistura.

O herbicida glyphosate sal de amônio só apresentou efeitos antagonísticos quando em mistura com haloxyfop no tocante ao controle

Tabela 3 - Médias de controle visual de amargoso aos 7, 14 e 28 dias após a aplicação (DAA) e porcentagem de redução de biomassa fresca em relação à testemunha, aos 28 DAA

\begin{tabular}{|c|c|c|c|c|}
\hline \multirow{2}{*}{ Tratamento } & \multicolumn{3}{|c|}{ Controle Visual (\%) } & \multirow{2}{*}{$\begin{array}{c}\text { Redução Biomassa } \\
\text { Fresca }(\%)\end{array}$} \\
\hline & 7 DAA & 14 DAA & 28 DAA & \\
\hline Glyphosate $^{1 /}$ & 31,3 bcde & $46,3 \mathrm{e}$ & $47,7 \mathrm{~d}$ & $63,7 \mathrm{abc}$ \\
\hline Glyphosate + Quizalofop & $46,3 \mathrm{ab}$ & $70,0 \mathrm{abc}$ & $78,8 \mathrm{c}$ & $78,9 \mathrm{a}$ \\
\hline Glyphosate + Haloxyfop & $27,5 \mathrm{de}$ & $63,8 \mathrm{bcd}$ & $76,3 \mathrm{c}$ & $69,0 \mathrm{abc}$ \\
\hline Glyphosate +Sethoxydim & 40,0 abcd & $75,0 \mathrm{abc}$ & $80,0 \mathrm{bc}$ & $71,0 \mathrm{abc}$ \\
\hline Glyphosate + Clethodim & $47,5 \mathrm{a}$ & $78,8 \mathrm{ab}$ & $81,3 \mathrm{abc}$ & $68,1 \mathrm{abc}$ \\
\hline Glyphosate $^{2 /}$ & 30,0 cde & $50,0 \mathrm{de}$ & $35,0 \mathrm{e}$ & $65,1 \mathrm{abc}$ \\
\hline Glyphosate + Quizalofop & $38,8 \mathrm{abcd}$ & $81,3 \mathrm{a}$ & $91,3 \mathrm{ab}$ & $73,2 \mathrm{abc}$ \\
\hline Glyphosate + Haloxyfop & $38,8 \mathrm{abcd}$ & $77,5 \mathrm{ab}$ & $80,0 \mathrm{bc}$ & $74,1 \mathrm{abc}$ \\
\hline Glyphosate + Sethoxydim & 40,0 abcd & $72,5 \mathrm{abc}$ & $78,8 \mathrm{c}$ & $69,6 \mathrm{abc}$ \\
\hline Glyphosate + Clethodim & $43,8 \mathrm{abc}$ & $65,0 \mathrm{bcd}$ & $82,5 \mathrm{abc}$ & $66,4 \mathrm{abc}$ \\
\hline Glyphosate $^{\frac{3}{}}$ & $40,0 \mathrm{abcd}$ & 61,3 cde & $68,8 \mathrm{~cd}$ & $56,4 \mathrm{c}$ \\
\hline Glyphosate + Quizalofop & $41,3 \mathrm{abcd}$ & $76,3 \mathrm{abc}$ & $91,0 \mathrm{a}$ & $72,0 \mathrm{abc}$ \\
\hline Glyphosate + Haloxyfop & 31,3 bcde & 61,3 cde & $76,3 \mathrm{c}$ & $64,6 \mathrm{abc}$ \\
\hline Glyphosate + Sethoxydim & 36,3 abcde & $68,8 \mathrm{abc}$ & $77,5 \mathrm{c}$ & $71,1 \mathrm{abc}$ \\
\hline Glyphosate + Clethodim & 30,0 cde & $68,8 \mathrm{abc}$ & $76,3 \mathrm{c}$ & $62,7 \mathrm{abc}$ \\
\hline Quizalofop & $28,8 \mathrm{cde}$ & $68,8 \mathrm{abc}$ & $82,5 \mathrm{abc}$ & $76,4 \mathrm{ab}$ \\
\hline Haloxyfop & $27,5 \mathrm{de}$ & $61,3 \mathrm{cde}$ & $78,8 \mathrm{c}$ & $79,6 \mathrm{a}$ \\
\hline Sethoxydim & 30,0 cde & $67,5 \mathrm{abc}$ & $75,0 \mathrm{c}$ & $63,1 \mathrm{abc}$ \\
\hline Clethodim & $22,5 \mathrm{e}$ & $61,3 \mathrm{de}$ & $68,8 \mathrm{~cd}$ & $60,5 b c$ \\
\hline $\mathrm{DMS}^{4 /}$ & 15,31 & 15,62 & 13,51 & 18,04 \\
\hline $\mathrm{F}$ & $22,21^{* *}$ & $9,37^{* *}$ & $57,49^{* *}$ & $3,24^{*}$ \\
\hline $\mathrm{CV}^{5 /}$ & 9,76 & 8,94 & 6,71 & 7,79 \\
\hline
\end{tabular}

는 Sal de isopropilamina; ${ }^{2}$ sal de amônio; ${ }^{3 /}$ sal potássico; ${ }^{4 /}$ diferença mínima significativa; ${ }^{5 /}$ coeficiente de variação; adição de óleo minera segundo recomendação do fabricante em todos os tratamentos. ${ }^{*},{ }^{* *}$ Médias seguidas pela mesma letra na coluna não diferem entre si pelo teste de Tukey em nível de probabilidade de erro de 5\%. 
visual, porém com todos os graminicidas quando foi avaliada a redução de biomassa fresca das plantas. Isso aconteceu porque o controle proporcionado pela mistura sempre foi muito próximo daquele obtido com o uso dos produtos isolados, e algumas vezes até inferior. O herbicida glyphosate sal potássico agiu de maneira aditiva em mistura com quizalofop em relação ao controle visual e em mistura com sethoxydim na redução de biomassa fresca. As outras associações foram antagonísticas (Tabela 4).

Em estádios mais avançados, o uso de misturas elevou o controle atingido pelo uso isolado de glyphosate. O uso de quizalofop foi superior ao uso de outros graminicidas em mistura. tendo sido melhor com o uso de glyphosate sal de amônio ou sal potássico. A interferência no processo de transferência de elétrons é mais rápida com a aplicação do glyphosate do que com um inibidor da ACCase (Araldi et al., 2011); somado a isso, o rápido efeito do glyphosate sal de isopropilamina pode ter diminuído a efetividade do herbicida quizalofop nesse caso.

O uso de haloxyfop e sethoxydim foi mais efetivo em misturas com glyphosate sal de isopropilamina e sal de amônio, se comparados com a aplicação isolada de glyphosate. O uso de isolado de graminicidas, com exceção de clethodim, também mostrou-se efetivo, e seu uso isolado pode ser alternativa para diminuir a aplicação do glyphosate, deixando de selecionar plantas resistentes a essa molécula. Outros trabalhos ressaltam, ainda, um elevado controle proporcionado pela aplicação isolada destes herbicidas (Fornarolli et al., 2011).

Tabela 4 - Médias de controle visual e porcentagem de redução de biomassa fresca de amargoso aos 28 dias após a aplicação (DAA) e relações entre misturas

\begin{tabular}{|c|c|c|c|c|c|c|}
\hline \multirow{2}{*}{ Tratamento } & Controle Visual (\%) & \multirow{2}{*}{$\mathrm{E}^{\mathrm{1}^{\prime}}$} & \multirow{2}{*}{$\mathrm{I}^{\underline{2} /}$} & \multirow{2}{*}{$\begin{array}{l}\text { Redução Biomassa } \\
\text { Fresca (\%) }\end{array}$} & \multirow{2}{*}{$\mathrm{E}$} & \multirow{2}{*}{ I } \\
\hline & $28 \mathrm{DAA}$ & & & & & \\
\hline Glyphosate $^{\frac{3}{}}$ & $53,8 \mathrm{D}$ & & & $63,7 \mathrm{abc}$ & & \\
\hline Glyphosate + Quizalofop & $78,8 \mathrm{C}$ & $92,0^{* *}$ & - & $78,9 \mathrm{a}$ & $91,4^{*}$ & - \\
\hline Glyphosate + Haloxyfop & $76,3 \mathrm{C}$ & $90,3^{* *}$ & - & $69,0 \mathrm{abc}$ & $92,7^{* *}$ & - \\
\hline Glyphosate +Sethoxydim & $80,0 \mathrm{Bc}$ & $88,5^{*}$ & - & $71,0 \mathrm{abc}$ & $86,2^{*}$ & - \\
\hline Glyphosate + Clethodim & $81,3 \mathrm{abc}$ & $87,5^{\mathrm{NS}}$ & $=$ & $68,1 \mathrm{abc}$ & $85,5^{* *}$ & - \\
\hline Glyphosate $^{4 /}$ & $35,0 \mathrm{E}$ & & & $65,1 \mathrm{abc}$ & & \\
\hline Glyphosate + Quizalofop & $91,3 \mathrm{Ab}$ & $88,8^{\mathrm{NS}}$ & $=$ & $73,2 \mathrm{abc}$ & $91,9^{* *}$ & - \\
\hline Glyphosate + Haloxyfop & $80,0 \mathrm{Bc}$ & $86,3^{* *}$ & - & $74,1 \mathrm{abc}$ & $92,8^{* *}$ & - \\
\hline Glyphosate + Sethoxydim & $78,8 \mathrm{C}$ & $84,0^{\mathrm{NS}}$ & $=$ & $69,6 \mathrm{abc}$ & $87,2^{* *}$ & - \\
\hline Glyphosate + Clethodim & $82,5 \mathrm{abc}$ & $79,7^{\mathrm{NS}}$ & $=$ & $66,4 \mathrm{abc}$ & $86,2^{* *}$ & - \\
\hline Glyphosate $^{\frac{5}{}}$ & $68,8 \mathrm{Cd}$ & & & $56,4 \mathrm{c}$ & & \\
\hline Glyphosate + Quizalofop & $91,0 \mathrm{~A}$ & $94,5^{\mathrm{NS}}$ & $=$ & $72,0 \mathrm{abc}$ & $89,6^{* *}$ & - \\
\hline Glyphosate + Haloxyfop & $76,3 \mathrm{C}$ & $93,2^{* *}$ & - & $64,6 \mathrm{abc}$ & $91,1^{* *}$ & - \\
\hline Glyphosate + Sethoxydim & $77,5 \mathrm{C}$ & $92,5^{* *}$ & - & $71,1 \mathrm{abc}$ & $83,7^{\mathrm{NS}}$ & $=$ \\
\hline Glyphosate + Clethodim & $76,3 \mathrm{C}$ & $90,5^{* *}$ & - & $62,7 \mathrm{abc}$ & $82,0^{* *}$ & - \\
\hline Quizalofop & $82,5 \mathrm{abc}$ & & & $76,4 \mathrm{ab}$ & & \\
\hline Haloxyfop & $78,8 \mathrm{C}$ & & & $79,6 \mathrm{a}$ & & \\
\hline Sethoxydim & $75,0 \mathrm{C}$ & & & $63,1 \mathrm{abc}$ & & \\
\hline Clethodim & $68,8 \mathrm{Cd}$ & & & $60,5 \mathrm{bc}$ & & \\
\hline $\mathrm{DMS}^{6 /}$ & $13,51 \mathrm{D}$ & & & 18,04 & & \\
\hline $\mathrm{F}$ & $23,97 \mathrm{C}$ & & & $3,24^{* *}$ & & \\
\hline $\mathrm{CV}^{-1 /}$ & 6,88 & & & 10,07 & & \\
\hline
\end{tabular}

${ }^{1 /}$ Valor Esperado (Colby); ${ }^{2 /}$ Interação (- antagonística; = aditiva); ${ }^{3 /}$ sal de isopropilamina; ${ }^{4 /}$ sal de amônio; ${ }^{5 /}$ sal potássico; ${ }^{6 /}$ diferença mínima significativa; ${ }^{1}$ coeficiente de variação; adição de óleo mineral segundo recomendação do fabricante em todos os tratamentos. ${ }^{*},{ }^{* *}$ Médias seguidas pela mesma letra na coluna não diferem entre si pelo teste de Tukey em nível de probabilidade de erro de 5\%. Ns Não significativo. 
O efeito antagonístico, na maioria dos casos, se deve ao fato de se esperar um controle efetivo com a associação dos produtos, que muitas vezes não ultrapassou o valor de $80 \%$.

Adegas et al. (2010), utilizando os herbicidas clethodim, fluazifop-p-buthyl, tepraloxydim, haloxyfop-methyl e paraquat, obtiveram controles superiores a 90\%. Entretanto, quando a planta cresce, ela rebrota, e esse controle cai para a média de 50\% (Procópio et al., 2006), confirmando que o controle de plantas perenizadas é mais dificil (Fornarolli et al., 2011).

A associação do glyphosate com haloxyfopmethyl ou sethoxydim foi também insatisfatória para um controle aceitável em outros trabalhos (Correia et al., 2010). Segundo Timossi (2009), um controle satisfatório é atingido pela aplicação isolada de nicosulfuron. Para Melo et al. (2012), o uso de misturas de glyphosate com graminicídas, como sethoxydim, haloxyfop-methyl, fluazifop-pbuthyl,fenoxaprop + clethodim e tepraloxydim, é uma alternativa viável. Estes autores chegaram à conclusão de que o uso isolado de clethodim é ineficiente no controle de plantas adultas.

Caso a planta esteja em pleno desenvolvimento vegetativo, talvez outras opções sejam mais eficientes que a aplicação desses tratamentos, como o uso de herbicidas de contato, que diminuem a área foliar da planta, o uso de roçagens anteriores à aplicação de herbicidas graminicidas ou o uso de aplicações sequenciais, como sugerido por outros autores (Melo et al., 2012). A utilização dessas estratégias é fundamental para evitar que ocorra uma seleção de plantas com resistência múltipla aos herbicidas inibidores da ACCase e inibidores da EPSPs, como aconteceu com plantas de azevém (Heap, 2013).

Assim, dos tratamentos utilizados, conclui-se que todos são eficazes, com exceção do uso de glyphosate isolado no manejo de plantas resistentes de Digitaria insularis, porém o controle deve ser feito em estádios iniciais de desenvolvimento da planta. Nesse estádio, a formulação glyphosate sal de isopropilamina foi superior às demais.
Em estádios mais desenvolvidos das plantas, o uso de quizalofop associado a glyphosate sal de amônio ou sal potássico promoveu os melhores controles (superiores a 90\%), porém a massa fresca das plantas não foi reduzida em mais de $80 \%$ em nenhum dos casos. Os herbicidas apresentaram efeitos aditivos se aplicados em estádios iniciais de desenvolvimento e antagonísticos na maioria dos casos de aplicação em estádios mais avançados. Neste caso, outras medidas de controle, somadas à associação de produtos, devem ser tomadas.

\section{AGRADECIMENTOS}

Ao CNPq e à FAPESP, pelo apoio aos pesquisadores, e à Cambuhy Agrícola, pela colaboração com o trabalho.

\section{LITERATURA CITADA}

ADEGAS, F. S. et al. Alternativas de controle químico de Digitaria insularis resistente ao herbicida glyphosate. In: CONGRESSO BRASILEIRO DA CIÊNCIA DAS PLANTAS DANINHAS, 2010, Ribeirão Preto. Resumos... Ribeirão Preto: Sociedade Brasileira da Ciência das Plantas Daninhas, 2010.

ARALDI, R. et al. Análise de fluorescência e consumo de água em Brachiaria decumbens após aplicação de glyphosate e haloxyfop-methyl. In: III SIMPÓSIO INTERNACIONAL SOBRE GLYPHOSATE: Uso Sustentável, 3., Botucatu. Anais... Botucatu: 2011. p. 324-329.

COLBY, S. R. Calculating synergistic and antagonistic responses of herbicides combinations. Weeds, v. 15, n.1, p. 20-22, 1967.

CORREIA, N. M. et al. Resposta de diferentes populações de Digitaria insularis ao herbicida glyphosate.

Planta Daninha, v. 28, n. 4, p. 769-776, 2010.

DUKE, S. O. Overview of herbicides mechanism of action. Env. Health Persp., v. 87, p. 263-271, 1990.

FORNAROLLI, D. A. et al. Manejo de biótipos de Digitaria insularis resistente ao herbicida glifosato. In: III SIMPÓSIO INTERNACIONAL SOBRE GLYPHOSATE: Uso Sustentável, 3., Botucatu. Anais... Botucatu: 2011. p. 317-320.

HARTZLER, B. Wich glyphosate products is best? Disponível em: <http:www.weeds.iastate.edu/ glyphosateformulations>. Acesso em: 11 jan. 2011. 
HEAP, I. The International Survey of Herbicide-R, 2012. Disponível em: <http://www.weedscience.com/summary/ home.aspx>. Acesso em: 1o abr. 2013.

LI, J. et al. Influence of formulation and glyphosate salt on absorption and translocation in three annual weeds. Weed Sci., v. 53, n.2, p. 153-159, 2005.

LICH, J. M.; RENNER, K. A.; PENNER, D. Interaction of glyphosate with postemergence soybean (Glycine max) herbicides. Weed Sci., v. 45, n.1, p. 12-21, 1997.

MELO, M. S. C. et al. Alternativas para o controle químico de capim-amargoso (Digitaria insularis) resistente ao glyphosate. R. Bras. Herbic., v. 2, n. 11, p. 195-203, 2012.

MELO, M. S. C. et al. Avaliação do crescimento dos biótipos de capim-amargoso (Digitaria insularis) resistente e suscetível ao glyphosate. In: CONGRESSO BRASILEIRO DA CIÊNCIA DAS PLANTAS DANINHAS, 28., 2010, Ribeirão Preto. Anais... Ribeirão Preto: 2010. p. 1187-1190.

MOLIN, W. T.; HIRASE, K. Effects of surfactants and simulated rainfall on the efficacy of the Engane formulation of glyphosate in johnsongrass, prickly sida and yellow nutsedge. Weed Biol. Manag., v. 5, p. 123-127, 2005.

MONSANTO. Produtos, herbicidas. Disponível em: <http:// www.monsanto.com.br/produtos/herbicidas/roundup/roundupready/roundup-ready.asp>. Acesso em: 15 out. 2013.

PROCÓPIO, S. O. et al. Efeitos de dessecantes no controle de plantas daninhas na cultura da soja. Planta Daninha, v. 24, n. 1, p. 193-197, 2006.
RODRIGUES, B. N.; ALMEIDA, F. S. Guia de herbicidas. 6.ed. Londrina: 2012. 697 p.

SHANER, D.; BRIDGES, D. Inhibitors of aromatic amino acid biosyntesis (glyphosate). In: Herbicide action course. West Lafayette: Purdue University, 2003. p. $514-529$.

SOCIEDADE BRASILEIRA DA CIÊNCIA DAS PLANTAS DANINHAS - SBCPD. Procedimentos para instalação, avaliação e análise de experimentos com herbicidas. Londrina: SBCPD, 1995. 42 p.

SILVA, F. M. L. et al. Eficiência fotossintética de Brachiaria decumbens após a aplicação de diferentes formulações de glyphosate. In: III SIMPÓSIO INTERNACIONAL SOBRE GLYPHOSATE: Uso sustentável, 3., Botucatu. Anais... Botucatu: 2011. p. 20-22.

STAKER, R. J.; OLIVER, L. R. Interaction of glyphosate with chlorimuron, fomesafen, imazethapyr and sulfentrazone. Weed Sci., v. 46, p. 652-660, 1998.

TIMOSSI, P. C. Manejo de rebrotes de Digitaria insularis no plantio direto de milho. Planta Daninha, v. 27, n. 1, p. 175-179, 2009.

VELINI, E. D. et al. Modo de ação do glyphosate. In: VELINI, E. D. et al. Glyphosate. Botucatu: Fundação de Estudos e Pesquisas Florestais, 2009. p. 113-133. 